\title{
A new design framework of the aesthetic data visualization
}

\author{
Qi Li \\ School of Art And Design \\ Shanghai University of Engineering Science \\ Shanghai, China \\ qili@sues.edu.cn
}

\author{
Chuanhong Xu \\ School of Art And Design \\ Shanghai University of Engineering Science \\ Shanghai,China \\ chuanghongxu@sina.com
}

\begin{abstract}
Data visualization is adopted as a visual tool identifying patterns for analyzing and evaluating complex data. Recent research demonstrates that aesthetics have potential to enhance emotional engagement in user perception of visual images. This paper examines the possibility of applied Chinese yijing aesthetic to unification of user's subject experience and objective data. Yijing presents a unity of the spirit of subject and the reality of object in Chinese traditional paintings, which is a unification of void and reality. This paper proposes a theoretical framework integrating aesthetic into data visualization to enhance uses' emotional engagement and data as creative media with the interactive technology that provides a general guidance of data visualization design.
\end{abstract}

Keywords- Data Visualization, Aesthetics, Yijing, Design, Creativity, Interactive technology

\section{INTRODUCTION}

Data visualization is used as a tool for communicating information effectively and efficiently through graphic representation (Friedman 2008). It aims to apply visual forms to display facts or patterns hidden inside abstract data. Those facts or patterns include natural phenomena or medical contexts, such as weather models or the structure of the cells. This process of data visualization emphasizes the functional and usability requirements for easy comprehension (Gaviria 2008). The forms of visualization initially used in communication purposes have 2D or 3D design, such as charts, graphs, waveform and 3D images. These visual forms share similarity that is recognition, readability and meaning. Those characteristics of form are moderated by the key criteria for effectiveness related to accuracy of communication and efficiency in terms of the speed at which meaning is extracted. Therefore, users may able to quickly and accurately recognize, read and understand the meaning of visualized data through the organized visual forms. Recent research demonstrated that aesthetic design of data visualization could enhance the human's perceive and will engage analysis more in the task (Cawthon \& Moere 2007).In addition to attractive data visualization, artists have adopted data or information as a new creative medium for their artistic practice. In the recently year, artists adopt data from external sources and collecting data as part of their artworks. It emerges as a concept of "beautiful data" represented as a visual image, which involves pleasing data patterns.

This paper therefore proposes a design conceptual framework that proposes a relationship along a continuum

DOI reference number: 10.18293/DMSVIVA2019-004. The project was supported by Shanghai IV Summit Discipline in Design and Shanghai Pujiang Talent Program. between communication, aesthetic experience and creative practice. The model demonstrates the relationship between the characteristics of communication, aesthetics and artistic expression, the typical forms, and how they are perceived by users. Based on this model, a new aesthetic design of data visualization that integrates the aesthetics of traditional Chinese yijing aesthetic and digital technology is proposed.

\section{AESTHETIC REPRESENTATIVE DATA}

Aesthetics in data visualization has been gaining attention as a means to promote a positive effect in viewers that enhances experience and amplifies the ability to interpret information. This has been recognized within the discipline of design, where research has demonstrated that visual attractiveness is a major factor in how people engage with content (Gaviria 2008). If data visualization has a high aesthetic quality, users may be encouraged to engage in a deep level of interpretation.

Research shows that aesthetic can promote the traditional focus of visualization on accuracy, efficiency and effectiveness (Card, Mackinlay \& Shneiderman 1999). At the same time, it can be used to prompt a subjective experience in the form of an emotional response. Tateosian, Healey and Enns (2007) emphasize a role for creativity in aesthetic visualization to engage users' attention in reaction to a stimulus. They argue that artistic techniques such as abstract representation and brush strokes techniques have been used to enhance aesthetic perception of visualization through non photo-realistic forms. Therefore, an aesthetic approach to visualization is quite different from traditional visualization methods. The traditional methods emphasize the perception of communication with speed and accuracy. This paper suggests that the Chinese yijing aesthetic has potential used to demonstrate this, enhancing the perception of experience by fusing subject and object and promoting pleasure and engagement.

\section{ChInESE YIJING AESTHETIC}

The notion of Yijing is a traditional Chinese aesthetic concept that refers to 'an exceptional state of mind' or 'an artistic conception' (Li 2009). It emphasizes the expression of an artist's inner emotion through representation of their subjective experience to the natural world (Zhang 2005). It suggests that yijing is similar to the Western concept of "empathy" that involves "the melding of the appreciating (or creating) self with the appreciated (or created) object" (Li 2009, 
p. 152). The phenomenon of "empathy" in Chinese aesthetics is the fusion of feeling and scene, which unifies self and object (Li 2009; Zhang 2005). The yijing aesthetic has been applied to literature, poetry, language, visual art and music (Liao 2011). In traditional Chinese painting, yijing constitutes criteria for the judgment of the value of the artworks (Liu \& Bralewskiz 2010). For example, a 'good' traditional Chinese painting usually possesses the yijing aesthetic quality, which is linking to a perfect melding of poem and image (Zong 2005). Yijing, therefore, can be considered as a means by which aesthetic media such as painting can be integrated into visualization to promote a positive subjective response aligned with its inherent values.

\section{PROPOSED CONCEPTUAL FRAMEWORK}

In Figure 1, a conceptual framework displays the relationship between the communication-based, aesthetic approaches and art practice to data visualization. It is argued that this model can be used to inform the design principles for data visualization. It could be a valuable design framework for design of data visualization depending on the different situation.

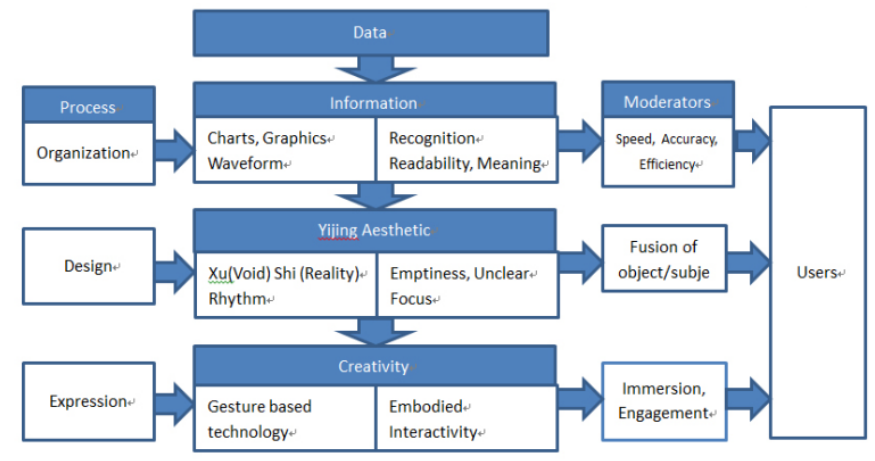

Figure 1. The proposed visualization conceptual framework

It is an axiomatic approach in that it represents a vertical data continuum, from data, through information, to aesthetics and to creativity. Each is a higher level of processing undertaken, initially through organization, through to design and then to expression. As the complexity of the data is reduced, the emotional experience of the user is enhanced, with the horizontal axis delineating the processes inherent in developing the three forms of representation on the left, with the user on the right. The interplay between the user and the representation is portrayed as factors that moderate or influence perceptions. While communication is primarily influenced by the need for speed, accuracy and efficiency, the user's experience is amplified by engaging with a more aesthetic representation. Therefore, the quality of the experience varies due to the nature of representation. Information presenting a clear way promotes understanding while aesthetics promote pleasure and engagement. Inevitably some relationship still exists between the need for communication and emotional engagement, though it would be expected that the objective communication elements may become increasingly ambiguous as a subjective emotional response is elicited from the user. The important factor in this framework is the differentiation between pure organization, design and expression as the main processes in generating the representation, with the latter process integrating aesthetic characteristics. Both information and aesthetics are defined by recognizable characteristics, though the forms may vary. In this framework, the yijing aesthetic achieves the specified characteristics of unity, harmony and so on through specific forms of reality, void and rhythm, unity, and the evocation of imagination of nature. Reality can be understood as tangible objects, which are represented as a realistic form with a focus on clarity. These 'real' objects constitute the most important parts in an image in terms of information. Void can be understood as emptiness, considered as the less important parts in an image. Void and reality have a complementary existence in visualization. This balance generates a visual rhythm that brings emotional harmony between subjectivity and objectivity, and evokes imagination.

While presented as information and emotional engagement, data can also be the media for art creativity. This model adopts gesture based technology to represent interactive data visualization. It suggests interacting with data engaging user's body building on yijing aesthetic characteristics focus on embodied interactivity, pleasure, harmony and balance through the complementarity of all elements rendered unreadable and unrecognizable. In addition, gesture based technology is used as an interactive technology for immersion and experience in the design of data visualization. As the visual representation of data is not recognized, it emphasizes that user's experience.

\section{Design of Aesthetic Visualization with the YiJING AESTHETIC}

As an instantiation of the theoretical framework proposed in Figure 1, a design has been undertaken that investigates the relationship between communication-focused waveforms and experience-focused yijing aesthetic. The paper applies sleep EEG data as source for visualization design. Sleep EEG data can be considered as a typical scientific data, which is seminal to human experience that has many connotations, such as divinatory or preposterous. As such, it forms an ideal medium for exploring aesthetics, particularly given the strong historical fascination with dreams or dream imagery within the Art disciplines (Shapiro, Sherman \& Kryger 2013). It has been designed as two stages as following:

\section{A. Approach to Design - Data as living objects}

Sleep EEG data, which has been visually represented in this work, is considered a living object that generates visual rhythm through data movement as lines rather than as static data that conveys information for comprehension (Figure 2). Indeed, the data is neither recognizable nor readable, and it does little to identify any valuable insights by its visual representation. In contrast, according to the proposed framework, the work emphasizes users' experience of a fusion between an individual's subjectivity and the so-called objective world of Western epistemology. The value of pleasure is generated through the design process. 


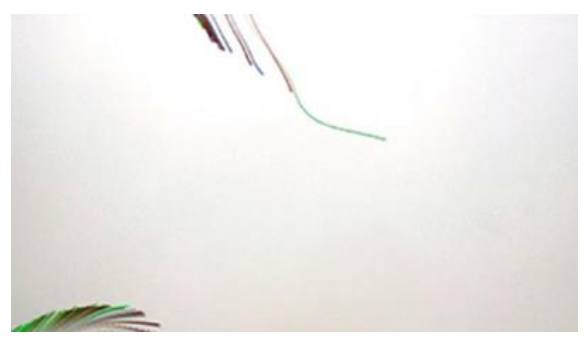

Figure 2. The screen shot of sleep EEG data visualization with Yijing aesthetic value

This design is an experimental work that specifically seeks to explore the evocation of the imagination through applying aesthetics to data visualization. As such, the focus is not primarily on efficiency of communication. Nevertheless, it is an accurate depiction of the data, which allows for recognition of the concept of sleep within a broader aesthetic interpretation. It is proposed that any deficit in accuracy and efficiency of communication is addressed through enhanced perception and sensation of experience.

\section{B. Approach to Expression - Data as performance}

This design work is based on the previous work, extending upon the engagement with embodied interactivity that was established by it. Gesture based technology was used to achieve this new aesthetic. Kinect is considered an ideal gesture-based technology with its distinct interactivity that enables one to "capture, track and decipher body movement, gestures and voice. The auditory and visual information serves as commands to interact with digital contents presented in games or software programs" (Hsu, 2011, p. 365). The work was inspired by Chinese Taoist health philosophy that emphasizes the practice of taijiquan performance with the flow of qi (void) moving through the body in order to achieve harmony and self-transcendence (Figure 3). The work is an outcome of this digital practice that attempts to represent sleep EEG data in the form of qi that users are able to interact with through an embodied data. This digital body is a kind of embodied digital body that unifies the user's body and data associated with the digital technology.

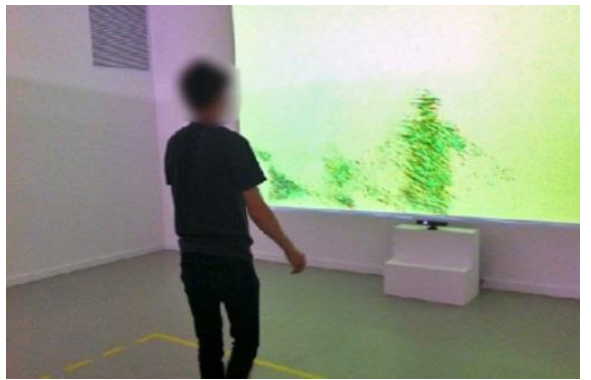

Figure 3. The screen shot of sleep EEG data visualization with interactive creativity using Kinect

\section{CONCLUSION}

The study explores how data, information and aesthetics operate as a communication continuum. It has been shown that visualization can enhance communication through organization with a focus on speed, efficiency and accuracy while current research has highlighted the role of aesthetics in integrating perception and sensation to create an emotional relationship that engages deeply with users and also approach to artistic creation in which data as medium. The conceptual framework described here promotes a new aesthetic approach to data visualization that has the potential to enhance the end user experience. By applying the form of the traditional Chinese yijing aesthetic to data visualization using sleep EEG data, a creative work has been developed that integrates traditional Chinese philosophy with a focus on void and reality, unity, rhythm and the evocation of imagination in terms of natural themes. By adopting gesture based technology to create embodied data visualization, an interactive work has been presented to a new interactive experience with data and technology. The visualization work will allow the forms and characteristics of information and aesthetics to be interrogated in a manner that identifies the key organizational and design principles required not just to communicate concepts but to create a heightened experience for users when they integrated into data visualization.

\section{ACKNOWLEDGMENT}

The project was supported by Shanghai IV Summit Discipline in Design and Shanghai Pujiang Talent Program.

\section{REFERENCES}

[1] SK. Card, JD. Mackinlay, \& Sneddon, I. N Readings in Information Visualisation: using vision to think, Academic Press, San Francisco, CA, USA.

[2] V. Jacobs, Data visualisation and infographics. viewed 2 September 2013,http://www.smashingmagazine.com/2008/01/14/mondayinspirationdatavisualisation-and-infographics, 2008.

[3] AR. Gaviria, 'When is information visualisation art? Determining the critical criteria', Leonardo, vol. 41, no. 5, pp. 479-482.

[4] H. J. Hsu, The potential of Kinect in education. International Journal of Information and Education Technology, 1(5), 365-370, 2011.

[5] Z. Li, The Chinese Aesthetic Tradition, University of Hawaii Press, Honolulu.2009.

[6] TL. Liao, Yijing: Mr. Wang meng-ou's Aesthetic of Language, vol. 25, no. 1, pp. 131-172. 2011.

[7] S. LIU, \& TW. BRALEWSKIZ, Chinese Landscape Inspiration "Yijing", viewed 10 March 2019, http://www.fem.uniag.sk/acta/download.php?id=1014

[8] CM.SHAPIRO, D. SHERMAN, \& MH. KRYGER, 'Sleep in art and literature', in MH Kryger (eds), Atlas of clinical sleep medicine, Elsevier Health Sciences, London. 2013.

[9] LG. TATEOSIAN, CG. HEALEY, \& JT. ENNS, 'Engaging viewers through nonphotorealistic visualisations', Paper presented at the Proceedings of the 5th international symposium on Non-photorealistic animation and rendering. 2007.

[10] W. ZHANG, The Inheritance and Contribution of Zong Huabai's Yijing Theory, viewed 16 April 2018 , http://www.kyoai.ac.jp/college/ronshuu/no-05/cho.pdf

[11] B. ZONG, A Walk with Aesthetics, Shanghai People's Press, Shanghai. 2005.

[12] MA. CARSKADON, \& WC, DEMENT, Qualities of Perceived Aesthetic in Data Visualisation, Paper presented at the Conference on Designing for User eXperiences (DUX'07), Chicago, Illinois, viewed 27 November 2018. 